\title{
Nichtstun kann das Risiko für Brustkrebs erhöhen
}

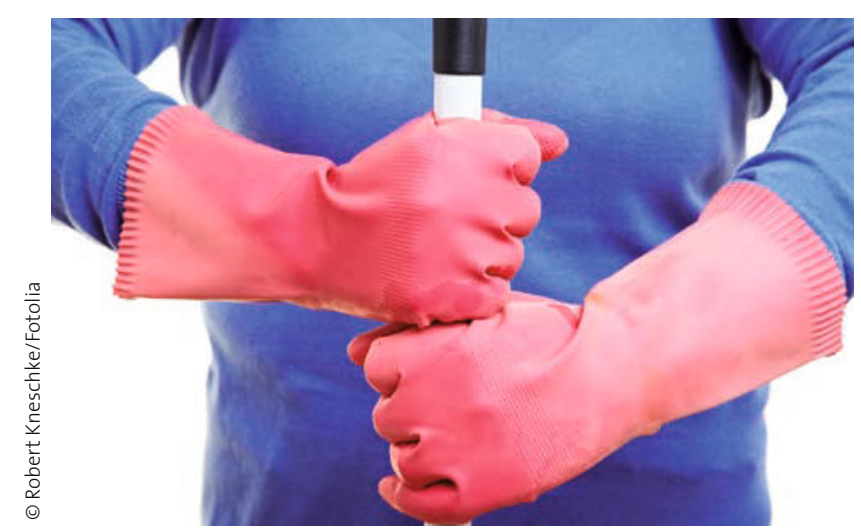

Brustkrebsprävention mit dem Schrubber.

TYP-2-DIABETES

\section{Ein Jahr Insulin, zwei Kilo mehr}

- Die Befürchtung vieler Typ-2-Diabetiker, nach dem Beginn einer Insulintherapie zuzunehmen, hat sich in einer Metaanalyse bestätigt. In der Untersuchung aus 51 randomisierten Parallelgruppenstudien mit 15202 Patienten lag die durchschnittliche Gewichtszunahme bei 2,1 kg im ersten Jahr der Therapie $(-0,85$ $\mathrm{kg}$ bis $+7,5 \mathrm{~kg}$ ). Die Zunahme war abhängig vom Insulinregime (mit einer basalen Therapie geringer als mit einer prandialen), der Therapiedauer, dem Ausgangs$\mathrm{HbA}_{1 \mathrm{c}}$ und seiner Veränderung sowie der Hypoglykämiefrequenz. Keinen Einfluss auf die Gewichtszunahme hatte das Ausgangskörpergewicht.

SPA =

- Miele l et al. Diabetologia 2010;53(Suppl 1) $\mathrm{S}_{23 .} \mathrm{OP} 4 \mathrm{O}$
— Nicht nur Freizeitaktivität, sondern auch die körperliche Mobilität und Anstrengung im (Arbeits-)Alltag kann das Risiko für invasiven Brustkrebs reduzieren. Das ist das Ergebnis einer großen Studie vom National Cancer Institute der USA. Für die Studie wurden Daten von 97000 postmenopausalen Frauen evaluiert. Die Studienteilnehmerinnen erhielten in den Jahren 1995/96 und 1996/97 jeweils zwei Fragebögen, in denen unter anderem Informationen zur körperlichen Aktivität im normalen Tagesablauf erhoben wurden. Bis zum Stichtag am 31.12.2003 entwickelten von diesen Frauen 2866 ein invasives Mammakarzinom und 570 einen In-situ-Brustkrebs.

Frauen, die sich tagsüber im Beruf oder im Haushalt körperlich betätigten, hatten gegenüber denen, die einen „sitzenden Lebensstil“ pflegten, ein relativ um $38 \%$ reduziertes Risiko für die Entwicklung eines invasiven Mammakarzinoms. Bei denen, die ihren Arbeitsweg über einen Zeitraum von zehn und mehr Jahren zu Fuß oder per Fahrrad zurücklegten, lag die relative Risikoreduktion gegenüber den „Bequemen“ bei 14\%. Der Einfluss auf In-situ-Karzinome war nicht signifikant.

$\mathrm{EH}=$

- Quelle: George SM et al. Am J Public Health 2010, Online-Publikation vom 23.9.2010, Doi:102105/AJPH.2009.180828

\section{VERDACHT AUF ERHÖHTES INFARKTRISIKO}

\section{Rosiglitazon wird vom Markt genommen}

- Die Europäische Arzneimittelbehörde EMA empfiehlt, die Zulassung für das Antidiabetikum Rosiglitazon aufzuheben. Das heißt, dass Avandia ${ }^{\circledR}$, Avandamet $^{\circledR}$ und Avaglim ${ }^{\circledR}$ in Europa demnächst nicht mehr erhältlich sein werden. Patienten, die bisher Rosiglitazon-haltige Medikamente einnehmen, sollten einbestellt und ihre Therapie entsprechend geändert werden.

Grundlage für die Entscheidung der EMA sind zwei neuere Studien, die die kardiovaskuläre Sicherheit von Rosiglitazon in Zweifel ziehen. Schon länger ist bekannt, dass das Glitazon Flüssigkeitsretention und Herzinsuffizienz begünstigen kann. Aus diesem Grund war die Substanz nur als Antidiabetikum der zweiten Wahl eingestuft und bei Diabetikern mit Herzinsuffizienz kontraindiziert. Seit Längerem gibt es außerdem Hinweise auf ein mög- licherweise erhöhtes Risiko für Herzinfarkte und Schlaganfälle. Dieser Verdacht wurde zwar entkräftet durch das Ergebnis der RECORD-Studie (nicht mehr kardiovaskuläre Ereignisse als mit Metformin oder Sulfonylharnstoff). Doch im Juni diesen Jahres wurden eine Registerstudie und eine Metaanalyse veröffentlicht, die unter Rosiglitazon einen Anstieg kardiovaskulärer Ereignisse (u.a. auch im Vergleich zu Pioglitazon) zeigen. Diese Studien veranlassten die EMA jetzt zu der Einschätzung, dass der Nutzen von Rosiglitazon nicht die damit verbundenen $\mathrm{Ri}$ siken aufwiegt. Zeitgleich mit der Entscheidung der EMA hat die US-amerikanische FDA beschlossen, den Einsatz von Rosiglitazon zwar nicht ganz auszusetzen, aber stark einzuschränken. Bs -

- Pressemitteilung der EMA, 23.9.2010 